\title{
Téoros
}

Revue de recherche en tourisme

\section{Pardon ? Vous avez bien dit : patrimoine immatériel montréalais?}

\section{Martin Drouin et Marie-Blanche Fourcade}

Volume 26, numéro 2, été 2007

URI : https://id.erudit.org/iderudit/1070950ar

DOI : https://doi.org/10.7202/1070950ar

Aller au sommaire du numéro

Éditeur(s)

Université du Québec à Montréal

ISSN

0712-8657 (imprimé)

1923-2705 (numérique)

Découvrir la revue

Citer cette note

Drouin, M. \& Fourcade, M.-B. (2007). Pardon ? Vous avez bien dit : patrimoine immatériel montréalais ? Téoros, 26(2), 74-76.

https://doi.org/10.7202/1070950ar d'utilisation que vous pouvez consulter en ligne.

https://apropos.erudit.org/fr/usagers/politique-dutilisation/ 


\section{Pardon? Vous avez bien dit: Patrimoine immatériel montréalais ?}

\section{Martin Drouin et Marie-Blanche Fourcade}

Le patrimoine de l'humanité s'est ouvert depuis quelques années à une nouvelle catégorie qui vise à circonscrire les chefs-d'œuvre du patrimoine oral et immatériel. Danses, langues, rites, musiques et savoir-faire côtoient désormais châteaux, cathédrales, pyramides, villes historiques, sites rupestres, parcs naturels et autres merveilles du monde. L'initiative tend, selon le directeur de l'Organisation des Nations Unies pour l'éducation, la science et la culture (UNESCO), Matsuura Koiricho, à élargir une " conception par trop restrictive » qui a «trop longtemps négligé [...] un pan entier du patrimoine culturel » (Koichiro, 2002). Les 90 chefs-d'œuvre actuellement proclamés - contre 830 biens matériels et naturels - cherchent à reconnaitre l'expression culturelle des peuples à travers des "pratiques, représentations, expressions, connaissances et savoir-faire - ainsi que les instruments, objets, artefacts et espaces culturels qui leur sont associés" (UNESCO, 2003). Ainsi, à une carte du patrimoine qui avait largement profité à l'Occident, avec l'Europe au premier plan, une nouvelle géographie se dessine pour donner davantage de place aux pays du Sud ${ }^{1}$. L'Amérique du Nord est encore, dans cette grande aventure, une terra incognita. Le patrimoine immatériel semble être pour les autres! Pas pour le nord de l'hémisphère, surtout pas pour les pays du nouveau continent.

L'inscription sur la liste unesquienne, dressée depuis l'adoption de la Convention sur le patrimoine mondial en 1972, a une influence considérable sur la visibilité des sites et, partant, sur le nombre de touristes attirés en ces lieux. Faire partie de ce club sélect constitue une formidable carte de visite. À Lunenburg en Nouvelle-Écosse, par exemple, plusieurs panneaux rappellent aux visiteurs qu'ils déambulent dans l'une des deux villes canadiennes inscrites sur la liste du patrimoine mondial. Depuis 1995, les campagnes publicitaires misent sur la qualité de l'offre qu'assure en quelque sorte le label. Si certains zélateurs du patrimoine s'inquiètent de la commercialisation de ces trésors, l'argent injecté dans l'entretien, la restauration et l'interprétation en favorise pourtant la sauvegarde. En sera-t-il de même de l'immatériel? L'importante couverture médiatique du carnaval de Binche en Belgique, l'une des rares manifestations européennes reconnues par l'UNESCO (en 2003), qui, depuis le Moyen Âge, marque le début du carême par des festivités de trois jours, permettrait de croire à un engouement similaire. II y aurait lieu toutefois de se demander s'il sera aussi facile d'apprécier le Gbofe d'Afounkaha

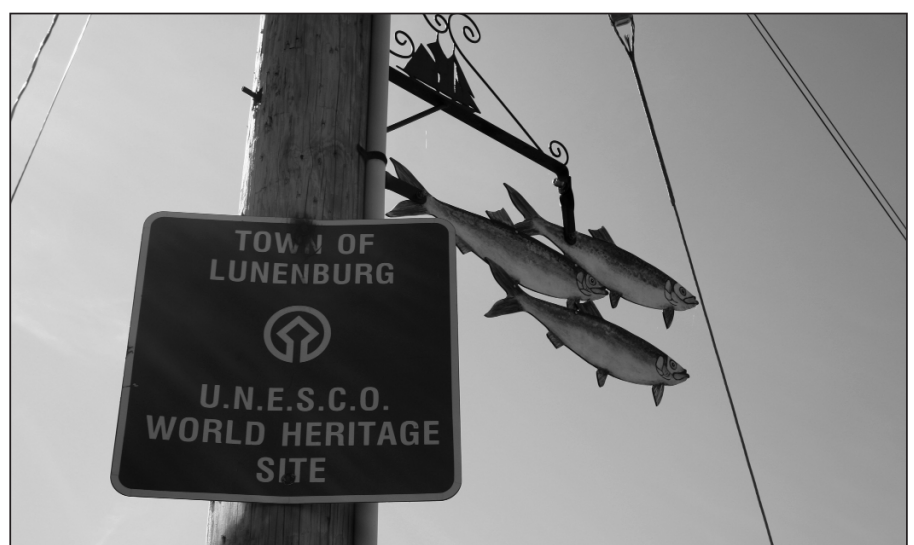

Des panneaux annoncent à Lunenburg, en Nouvelle-Écosse, que la ville est inscrite sur la liste du patrimoine mondial de I'UNESCO.

Photo : Martin Drouin

en Côte d'Ivoire ou de participer au carnaval d'Oruro en Bolivie que de se rendre dans cette petite ville du Hainaut, située à une soixantaine de kilomètres au sud de Bruxelles. Serait-il possible de détourner les touristes tentés d'aller admirer les dessins sur le sable du Vanuatu dans le Pacifique Sud vers des villes canadiennes pour leur faire goûter des manifestations de l'immatériel, a fortiori dans une métropole de deux millions d'habitants?

La question semble presque incongrue. Lorsqu'on évoque la problématique du patrimoine immatériel au Québec et au Canada, le premier élan est plutôt de se tourner vers le monde rural. En effet, celui-ci serait davantage porteur de savoir-faire et de coutumes. Musique traditionnelle, contes transmis au fil des générations, métiers anciens y trouveraient un terreau fertile pour survivre ou y être ranimés en toute légitimité. Les premiers folkloristes intéressés par les arts et les traditions populaires ne s'y sont pas trompés. C'est vers les campagnes qu'ils se sont lancés pour recueillir et observer, calepin en main, des pratiques qu'ils croyaient appartenir à un autre temps et même à une civilisation en voie de disparition. Bien sûr, la situation a évolué. II n'empêche que le temps des sucres, par exemple, avec la forte médiation touristique dont il bénéficie, est l'une des manifestations les plus citées pour témoigner de cette continuité (Bouliane, 2007; LaFerrière, 2007). Malgré des transformations manifestes et l'amalgame des pratiques que l'on peut désormais y trouver, l'incontournable visite à la cabane à sucre se rangerait dans le champ de l'immatériel à classer parmi un patrimoine à conserver. La ville, quant à elle, trop occupée à consolider 
son développement, à se soumettre aux diktats de l'économie mondiale et à absorber de nouvelles populations, n'aurait pas grand-chose à offrir. Le patrimoine immatériel semble être encore ici pour les autres! Pas pour la ville, surtout pas pour Montréal.

Adoptée par les autorités municipales montréalaises en 2005, la politique du patrimoine nous apprend pourtant le contraire. Alors que le champ de l'immatériel était absent du généreux panorama dressé en 1998 par Serge Careau et Perla Serfaty, voilà qu'il est défini aujourd'hui comme une «référence identitaire pour les Montréalais » (Montréal, 2005 : 76). Tout comme l'environnement bâti et naturel, il contribuerait à forger la spécificité de Montréal. La politique montréalaise présente d'ailleurs le patrimoine culturel immatériel comme :

[...] le produit évolutif d'un patrimoine souvent issu de milieux ruraux d'ici et d'ailleurs, mais transformé par l'histoire, le contexte social et économique, les événements, l'industrialisation et l'urbanisation propres à Montréal. II est l'héritage des peuples fondateurs de Montréal, des groupes et des communautés qui ont construit, habité et surtout marqué la ville. Il est en constante évolution et se nourrit des courants historiques, religieux, sociaux et culturels qui traversent la culture urbaine. II est transmis grâce à des porteurs, des acteurs, des praticiens et grâce aux citoyens de Montréal. (Montréal, 2005 : 17)

Partant d'une telle définition, il faut se demander comment ce patrimoine immatériel se manifeste en réalité et à l'esprit de ceux qui visitent et vivent la ville.

Spontanément, le patrimoine immatériel montréalais serait associé aux communautés ethnoculturelles. Plusieurs raisons peuvent l'expliquer, notamment le fait d'avoir l'impression que les urbains ne peuvent être porteurs de traditions et que, pour le déceler, il faudrait nécessairement regarder ailleurs. II est vrai aussi que les communautés culturelles se sont «réenracinées » dans la ville en portant fièrement les marques de leur identité. Parmi les groupes culturels recensés à Montréal ${ }^{2}$, certains sont plus visibles que d'autres, notamment en raison de leur ancrage territorial marqué (soit à la suite de leur longue implantation, soit par leur forte concentration). Les portes monumentales en forme d'arc de triomphe, qu'elles soient chinoises ou italiennes, invitent les badauds, les touristes ou les résidants à entrer dans un autre univers marqué par l'ailleurs qui fait dire à chacun : "On se croirait en Chine ou en Italie. "Par ces traces évidentes dans le paysage, la rencontre avec le patrimoine de la communauté est d'autant plus facile qu'elle est balisée. Elle se pratique dans le quotidien par la fréquentation de commerces ou de restaurants, telle l'artère Jean-Talon avec ses nombreux restaurants indiens, mais aussi dans l'événementiel, avec les fêtes qui animent les quartiers dont le Nouvel An chinois est un exemple. Alors qu'ils sont peu visibles dans l'ensemble patrimonial québécois matériel, bâti ou artefactuel, les migrants se sont davantage démarqués par leurs traditions orales, leurs fêtes et leur gastronomie. Ce sont d'ailleurs ces patrimoines tangibles et intangibles - glissés dans les valises lors du départ - qui, métissés de génération en génération, font aujourd'hui la spécificité des communautés ou autour desquels tout au moins elles se rassemblent.
En dehors des événements liés à chaque communauté ou à la vie de quartier, plusieurs manifestations permettent de donner une voix - et une visibilité - à des groupes culturels peu ou moins connus que d'autres. Tel est le cas des Folklories, qui se sont tenues pour la première fois en novembre 2004, dont les objectifs étaient de faire connaître et de transmettre le patrimoine immatériel des communautés qui façonnent Montréal ainsi que de partager la diversité dans le cadre d'une grande fête. Ce sont, en effet, plus d'une soixantaine d'associations qui ont installé leur kiosque et organisé des activités et des spectacles pour partager leur culture, leurs traditions et leurs

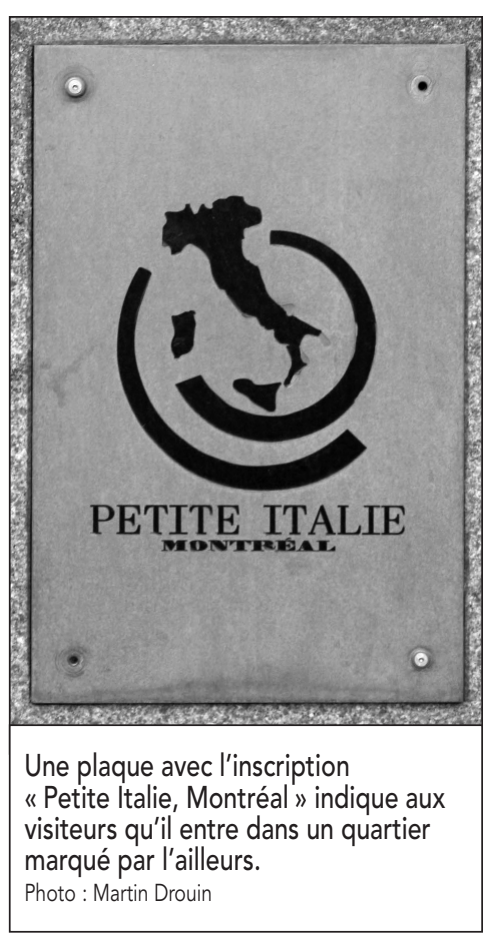
savoir-faire. Dépassant le caractère ponctuel de cette activité menée conjointement par la Société du patrimoine d'expression au Québec, la Ville de Montréal, le ministère de la Culture et des Communications et l'arrondissement Villeray-Saint-Michel-ParcExtension, d'autres événements tentent de s'inscrire dans un temps plus long et un public élargi. En ce sens, une autre initiative, venue du Centre d'histoire de Montréal, a fait découvrir aux visiteurs, par le biais d'une série d'expositions en 2003-2004, l'histoire de trois communautés : les Syriens-Libanais, les Portugais et les Haïtiens ${ }^{3}$. Bien plus que des expositions, ces événements sont les fruits d'un projet de conservation des mémoires communautaires intitulé les "Cliniques de mémoire », qui a pour but de faire la collecte de témoignages, d'objets et de photographies qui retracent la vie des migrants et de permettre, par le biais des échanges entre les jeunes qui enregistrent ou inventorient et les plus âgés qui racontent, de tisser des liens intergénérationnels et intracommunautaires. Ainsi, que ce soit à l'échelle de la cité, par la prise de conscience d'une diversité, ou que ce soit au coeur des groupes, par la compréhension de la fragilité du patrimoine immatériel et d'une responsabilité à l'entretenir, on assiste à une véritable reconnaissance d'une culture des autres au sein de l'espace partagé qu'est Montréal.

Le patrimoine immatériel montréalais ne serait-il que celui des autres transposés dans la métropole? Ne serait-ce pas une erreur que de s'en tenir à l'ailleurs pour trouver des manifestations de l'intangible? II semblerait que oui, surtout lorsqu'on se tourne vers l'univers des associations et des groupes qui travaillent à la conservation, à la diffusion et à la transmission du patrimoine d'expression. Le calendrier des manifestations culturelles montréalaises est en effet jalonné d'événements qui proposent aux citadins de se replonger dans leurs traditions. Les Veillées du Plateau, qui se tiennent tous les troisièmes samedis du mois au Centre du Plateau, rendent accessible au plus grand nombre un répertoire de musiques et de danses traditionnelles québécoises dans le but de les faire connaître et de les conserver. Dans un autre registre, la 


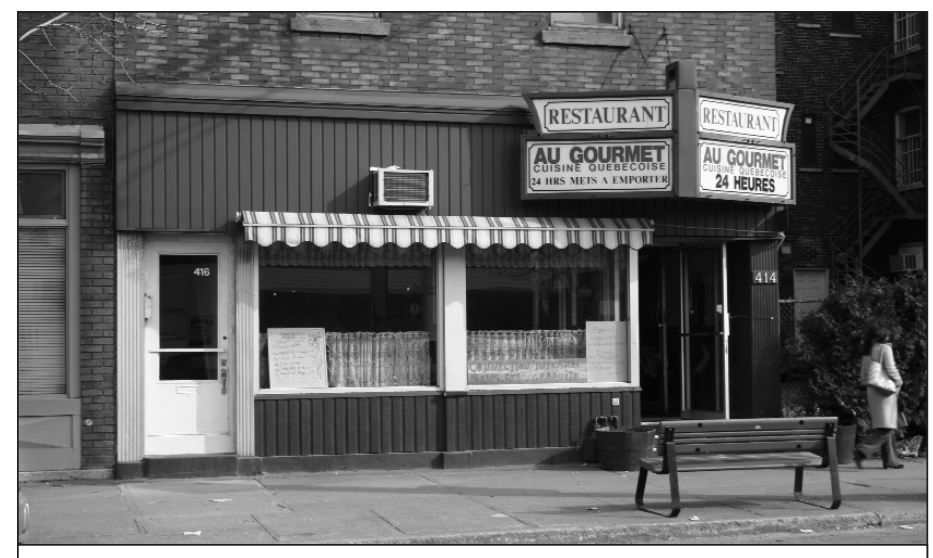

Le restaurant "Au Gourmet », qui propose une cuisine québécoise, n'est pas à première vue associé à une tradition culinaire à classer dans le patrimoine immatérielle.

Photo: Martin Drouin

fête de la Saint-Jean-Baptiste avec sa parade et ses spectacles est un moment privilégié de l'année pour célébrer l'appartenance québécoise et pour reproduire des gestes festifs portés par des parents ou des grands-parents, qu'ils soient de portée religieuse ou patriotique. Passées de telles manifestations publicisées à l'échelle de la ville ou des milieux spécialisés, il ne faudrait pas omettre de parler des savoir-faire qui forment la trame des métiers et des habiletés propres à la ville. L'inventaire des ressources ethnologiques du patrimoine immatériel en cours de réalisation par les chercheurs associés à la Chaire de recherche du Canada en patrimoine ethnologique en a dégagé quelques-uns qui annoncent un chantier des plus intéressants : fabricant de valise, tailleur, joaillier, sculpteur, chocolatier et glacier, sans oublier le Marché Jean-Talon. Les fêtes, les traditions ou les savoir-faire seraient orphelins si l'on n'accolait pas à leur existence les mémoires du quotidien qui façonnent Montréal. L'Action terroriste socialement acceptable (ATSA) a justement mis sur pied un projet qui permet de découvrir ces mémoires urbaines. Intitulé le "FRAG sur la Main », le projet consiste en l'installation d'une série de 32 panneaux à des endroits stratégiques du boulevard Saint-Laurent, témoins des multiples strates historiques, sociales et culturelles qui ont fait la vie de l'artère. En plus des compositions graphiques, les passants peuvent aussi télécharger une baladodiffusion qui complète le parcours visuel $^{4}$. Ce ne sont donc pas uniquement les bâtiments qui sont pris en compte et évalués, mais aussi et surtout la vie des individus. Les porteurs de mémoire ont, en ce sens, trouvé une place de choix au cœur du Musée de la personne, hébergé au Centre d'histoire de Montréal. Le slogan "Vous faites partie de l'histoire » atteste bien de l'importance que l'institution virtuelle accorde aux témoignages considérés comme la collection du musée.

Loin d'être une incongruité, la valorisation de l'immatériel s'inscrit dans la formidable expansion de la notion de patrimoine à Montréal. Elle est en quelque sorte une suite logique aux luttes menées depuis plusieurs décennies. Des monuments historiques aux quartiers à conserver, le cadre bâti s'est progressivement transformé en un cadre de vie signifiant. Ce n'est plus seulement l'ensemble des bâtiments, des rues, des places, des parcs et des jardins qui singulariserait la métropole, mais les Montréalais eux-mêmes qui l'habitent, l'animent et lui donnent vie (Drouin, 2005). C'est ce que le succès des œuvres comme les Chroniques du Plateau Mont-Royal de Michel Tremblay (1978-1989) ou Le Matou d'Yves Beauchemin (1981) avait, d'une certaine manière, célébré : l'ordinaire montréalais. L'esplanade de la place des Arts n'est pas la place Jeema el-Fna au Maroc et le tam-tam sur le mont Royal n'a pas de tradition bimillénaire comme le Kutiyattam en Inde. II n'empêche que des petits riens de l'ordre du banal qui font tout autant l'atmosphère que l'histoire sociale de la ville pourraient être qualifiés de "petit patrimoine immatériel ». Le «je-ne-sais-quoi » montréalais qui frappe bien des observateurs de passage dans la ville pourrait dès lors s'inscrire dans cette évolution. Reste à savoir comment en faire, s'il telle chose est possible, une expérience touristique propre à la métropole... à moins d'avoir peur que cette atmosphère montréalaise se folklorise.

Martin Drouin est professeur associé au département d'études urbaines et touristique de l'Université du Québec à Montréal et Marie-Blanche Fourcade est coordonnatrice de l'Institut du patrimoine à la même université.

\section{Notes}

1 Pour une réflexion sur l'évolution de la notion de patrimoine immatériel, on peut se référer à Kirshenblatt-Gimblett (2004) et à Jadé (2006).

2 Au recensement de 2001, plus de 90 origines ethniques différenciées étaient recensées à Montréal (Statistiques Canada, 2001).

3 Pour des détails sur les expositions réalisées depuis l'ouverture du musée en 1983, consulter le site Internet du Centre d'histoire de Montréal (2007)

4 Pour des détails et pour télécharger la baladodiffusion, voir le site Internet officiel de l'ATSA (2006).

\section{Bibliographie}

ATSA (2006), «FRAG sur la main », [http://www.atsa.qc.ca/pages/frags2ac cueil.asp], (consulté le 5 avril 2007).

Beauchemin, Yves (1981), Le Matou, Montréal, Québec/Amérique, 583 p.

Bouliane, Martine (2007), " 10 cabanes à sucre », La Presse, 3 mars, cahier Vacances-Voyages, p. 4.

Careau, Serge et Perla Serfaty (1998), Le patrimoine de Montréal. Document de référence, Montréal, Ville de Montréal / Ministère de la Culture et des Communications, $168 \mathrm{p}$.

Centre d'histoire de Montréal (2007), «Expositions passées », [http://ville.mon treal.qc.ca/portal/page?_pageid=2497,3089837\&_dad=portal\&_sche ma=PORTAL], (consulté le 5 avril 2007).

Drouin, Martin (2005), Le combat du patrimoine à Montréal (1973-2003), Québec, Presses de I'Université du Québec, 386 p.

Jadé, Mariannick (2006), Le patrimoine immatériel : perspectives d'interprétation du concept de patrimoine, Paris, L'Harmattan, $277 \mathrm{p}$.

Kirshenblatt-Gimblett, Barbara (2004), «Intangible Heritage as Metacultural Production », Museum International, vol. 56, nos 1-2, p. 52-65.

Koichiro, Matsuura (2002), «Éloge du patrimoine culturel immatériel », Le Monde, 11 septembre, p. 15.

LaFerrière, Michèle (2007), “Comme au chalet : L'Érablière Le Chemin du Roy fait table rase de la 'cabane à Ti-Guy' », Le Soleil, 17 mars, p. M17.

Montréal, Ville de (2005), Politique du patrimoine, Montréal, Ville de Montréal, 97 p.

Statistiques Canada (2001), «Origines ethniques, Montréal (QC) », n’ 97F0024XIF2001006, [http://www12.statcan.ca/english/census01/pro ducts/highlight/ETO/Table1.cfm?Lang=F\&T=501\&GV=2\&GID=462], (consulté le 5 avril 2007).

Tremblay, Michel (1978-1989), Chroniques du Plateau Mont-Royal, Montréal, Leméac, 5 vol.

UNESCO (2003), Convention pour la sauvegarde du patrimoine, [http://por tal.unesco.org/fr/ev.php-URL_ID=17716\&URL_DO=DO_TOPIC\&URL_ SECTION=201.html], (consulté le 5 avril 2007). 